TECHNICAL NOTE

\author{
N. Ma \\ X. Lou \\ T.-Q. Zhao \\ E.-H.-C. Wong \\ W.-J. Jiang
}

\section{Intraobserver and Interobserver Variability for Measuring the Wall Area of the Basilar Artery at the Level of the Trigeminal Ganglion on High-Resolution MR Images}

SUMMARY: We evaluated the intraobserver and interobserver variability for WA measurement of an atherosclerotic BA by using HRMRI. Ten consecutive patients underwent HRMRI, and the WA of the BA at the level of the trigeminal ganglion was measured by 2 independent readers and repeated by 1 reader 1 month later. There was strong agreement for intraobserver and interobserver measurements in the ICC and by the Bland-Altman method. Measurement of the WA in an atherosclerotic BA by HRMRI was reproducible.

ABBREVIATIONS: $\mathrm{BA}=$ basilar artery; $\mathrm{Cl}=$ confidence interval; $\mathrm{ETL}=$ echo-train length; $\mathrm{FSE}=$ fast spin-echo; HRMRI = high-resolution MR imaging; ICC = intraclass correlation coefficient; PDWI = proton attenuation-weighted imaging; $\mathrm{T} 1 \mathrm{WI}=\mathrm{T} 1$-weighted imaging; $\mathrm{T} 2 \mathrm{WI}=\mathrm{T} 2$-weighted imaging; $\mathrm{TIA}=$ transient ischemic attack; TOF = time of flight; $\mathrm{WA}=$ wall area
$\mathbf{P}$ laque burden, represented by WA, has substantial usefulness in the assessment of atherosclerosis. ${ }^{1}$ Imaging modalities used to measure plaque burden of the aorta and coronary, carotid, and peripheral arteries include intravascular sonography, ${ }^{2}$ multidetector $\mathrm{CT},{ }^{3} \mathrm{~B}$-mode sonography determination of arterial intima media thickening, ${ }^{4}$ and MR imaging. ${ }^{5}$

HRMRI has advantages over other modalities in depicting the wall of the BA because it is noninvasive and radiation-free and lacks bone artifacts in the posterior fossa. ${ }^{6,7}$ It also provides excellent boundary definition for BA-CSF and bloodintima borders, which allows a more precise measurement of the WA of the BA. However, to our knowledge, the reproducibility of such measurements by HRMRI has not been addressed. Our goal was to evaluate the intraobserver and interobserver variability of WA measurements of an atherosclerotic BA by using HRMRI.

\section{Materials and Methods}

\section{Subjects}

This prospective cohort study was approved by our institutional ethics committee, and written informed consent was obtained before HRMRI examination. Eligible patients had the following characteristics: 1) ischemic stroke or TIA of the posterior circulation within 90 days, 2) BA stenosis $\geq 70 \%$ on conventional angiography, and 3 ) $\geq 2$ atherosclerotic risk factors (hypertension, hyperlipidemia, diabetes

Received October 31, 2009; accepted after revision January 12, 2010.

From the Department of Neurology and Interventional Neuroradiology (N.M., W.-J.J.), Beijing Tiantan Hospital, Capital Medical University, Beijing, China; Department of Radiology (X.L., T.-0.Z.), PLA General Hospital, Beijing, China; and Department of Medicine and Therapeutics (E.-H.-C.W.), Chinese University of Hong Kong, Hong Kong, China.

This study was supported by the National Natural Science Foundation of China (30670731) and PLA 11 5th Medical Foundation (060070)

Please address correspondence to W.-J. Jiang, MD, Department of Neurology and Interventional Neuroradiology, Beijing Tiantan Hospital, Capital Medical University, No. 6 Tiantan Xili, Beijing 100050, China; e-mail: cjr.jiangweijian@vip.163.com

Indicates open access to non-subscribers at www.ajnr.org

DOI 10.3174/ajnr.A2049 mellitus, cigarette smoking, and obesity). Hyperlipidemia was defined as a total cholesterol level $\geq 240 \mathrm{mg} / \mathrm{dL}$, low-attenuation lipoprotein cholesterol level of $\geq 160 \mathrm{mg} / \mathrm{dL}$, or current use of medication for lowering blood cholesterol levels.

We excluded patients with following conditions: 1) arteritis, 2) claustrophobia or contraindications to MR imaging such as the presence of ferromagnetic implants, 3) medically unable to remain in the MR imaging scanner for $>40$ minutes, and 4$)<2$ atherosclerotic risk factors.

\section{MR Imaging Protocol}

Cross-sectional imaging was performed with an 8-channel phasedarray head coil in a 3T scanner (Signa, TwinSpeed 3T; GE Healthcare, Milwaukee, Wisconsin). 3D TOF images were obtained with TR/ $\mathrm{TE}=21 / 3.2 \mathrm{~ms}, \mathrm{FOV}=16 \times 16 \mathrm{~cm}$, thickness $=1 \mathrm{~mm}$, matrix $=$ $256 \times 256$, and NEX $=1$. Then, PDWI and T2WI of 12-14 sections and T1WI of 4 sections precontrast and postcontrast were acquired, which included images at the level of trigeminal ganglion. If the BA was severely tortuous, the scan plane was angled to ensure that images acquired were perpendicular to the BA. Parameters for PDWI and T2WI were as follows: FSE, TR $=3000 \mathrm{~ms}, \mathrm{TE}=12.5 \mathrm{~ms}$ for PDWI and $\mathrm{TE}=62.3 \mathrm{~ms}$ for $\mathrm{T} 2 \mathrm{WI}, \mathrm{FOV}=16 \mathrm{~cm} \times 16 \mathrm{~cm}$, thickness $=$ $2 \mathrm{~mm}$, matrix $=256 \times 320, \mathrm{NEX}=2, \mathrm{ETL}=10$. Flow compensation and an inferior spatial saturation band were used to suppress the pulsation artifacts induced by inflow blood. T1WI was obtained by double inversion recovery FSE with the following parameters: TR/TI/ $\mathrm{TE}=800 / 650 / 8.6 \mathrm{~ms}, \mathrm{FOV}=16 \times 16 \mathrm{~cm}$, thickness $=2 \mathrm{~mm}$, matrix $=256 \times 320, \mathrm{NEX}=4, \mathrm{ETL}=12$. Postcontrast T1WI was acquired 5 minutes after gadolinium injection $(0.1 \mathrm{mmol} / \mathrm{kg}$ of gadopentetate dimeglumine, Magnevist; Bayer Schering Pharma, Berlin,

ICCs and lower and upper limits of the $95 \% \mathrm{CI}$ for intraobserver and interobserver variability

\begin{tabular}{lcc}
\hline & \multicolumn{2}{c}{ ICC $(95 \% \mathrm{CI})$} \\
\cline { 2 - 3 } & Intraobserver & Interobserver \\
\hline T1WI & $0.973(0.893-0.993)$ & $0.981(0.923-0.995)$ \\
PDWI & $0.976(0.901-0.994)$ & $0.957(0.827-0.990)$ \\
T2WI & $0.973(0.892-0.993)$ & $0.962(0.847-0.991)$ \\
Postcontrast T1WI & $0.980(0.918-0.995)$ & $0.989(0.956-0.997)$ \\
\hline
\end{tabular}


Germany). The cross-sectional voxel size was $0.6 \times 0.6 \mathrm{~mm}$ on TOF and $0.6 \times 0.5 \mathrm{~mm}$ on T1WI, T2WI, and PDWI. Fat suppression was used to reduce the signal intensity from surrounding fatty tissues. A zip 512 matrix technique was used to enhance spatial resolution.

\section{Measurement of the WA of the BA}

An image-quality rating ( $1=$ poor, 2 = adequate, $3=$ good $)$ was performed by a radiologist (X.L.) for each image. Patients with poor images due to subject motion or low signal intensity-to-noise ratio were excluded. The BA cross-sectional area at the level of trigeminal ganglion was measured by readers blinded to the clinical presenta- tion, by using research software (FuncTool 2 software, GE Healthcare) on the ADW4.3 workstation (Sun Microsystems, Santa Clara, California). The BA-CSF border was used to manually trace the vessel area and the blood-intima boundary for the lumen area. The WA was calculated as the difference between vessel area and lumen area.

To assess intraobserver variability, reader 1 (X.L.) measured the WA at the level of trigeminal ganglion, with the second set of measurements performed 1 month later. To examine interobserver variability, reader 2 (N.M.) determined the WA at the same level and compared the measurements with the first set obtained by reader 1 .
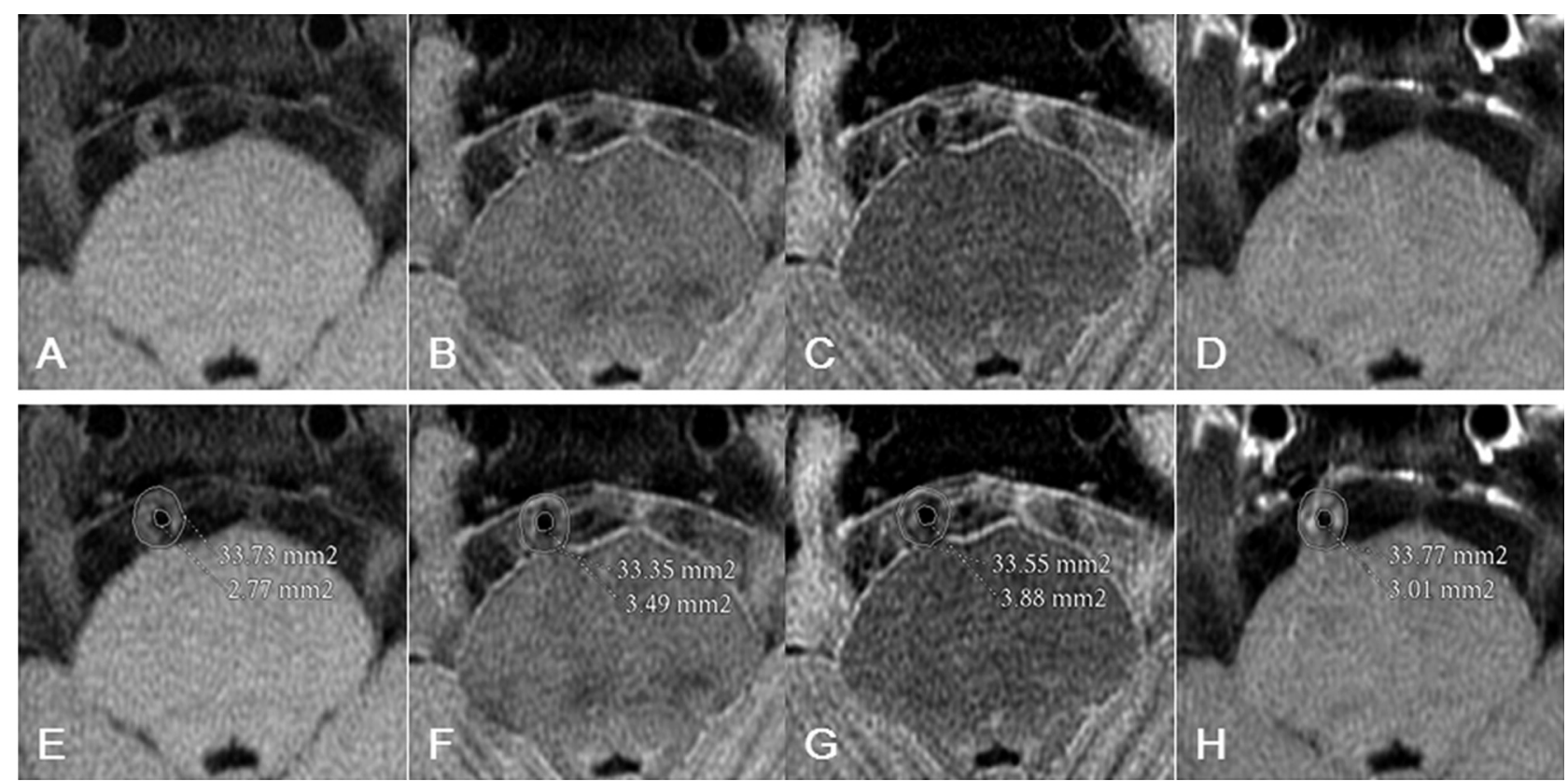

Fig 1. A 48-year-old man with hypertension, hyperlipidemia, and smoking history presented with recurrent transient attacks of dizziness and diplopia for 10 months. A, At the level of trigeminal ganglion, T1WI shows slight hyperintense eccentric wall thickening of the BA compared with the temporal muscle (not shown). PDWI $(B)$ and T2WI $(C)$ show isointense vesse wall thickening. $D$, Postcontrast T1WI shows heterogeneous enhancement, suggesting that the atherosclerotic plaque was composed of a lipid-rich necrotic core. The BA-CSF border and the blood-intima border can be demarcated on T1WI $(A)$, PDWI $(B)$, T2WI $(C)$, and postcontrast T1WI $(D)$. The outlines of the BA lumen and outer wall boundary are shown on T1WI $(E)$, PDWI $(F)$, T2WI $(G)$, and postcontrast T1WI $(H)$.
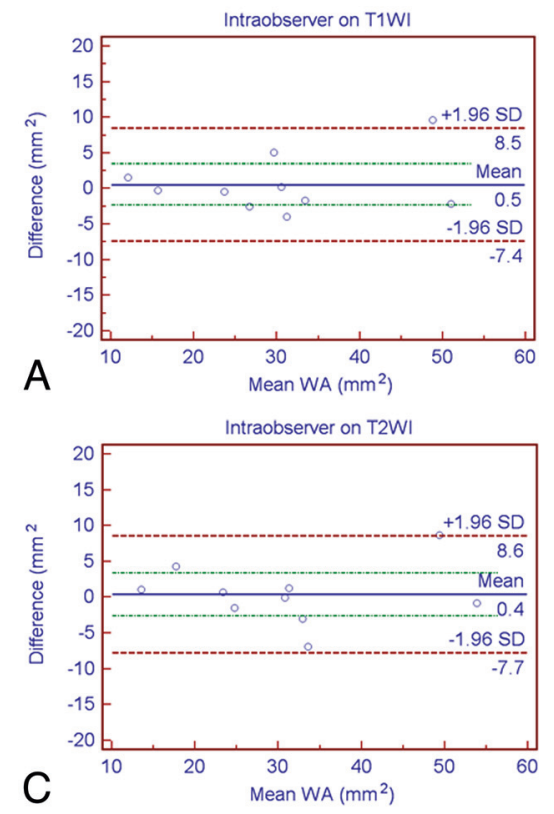
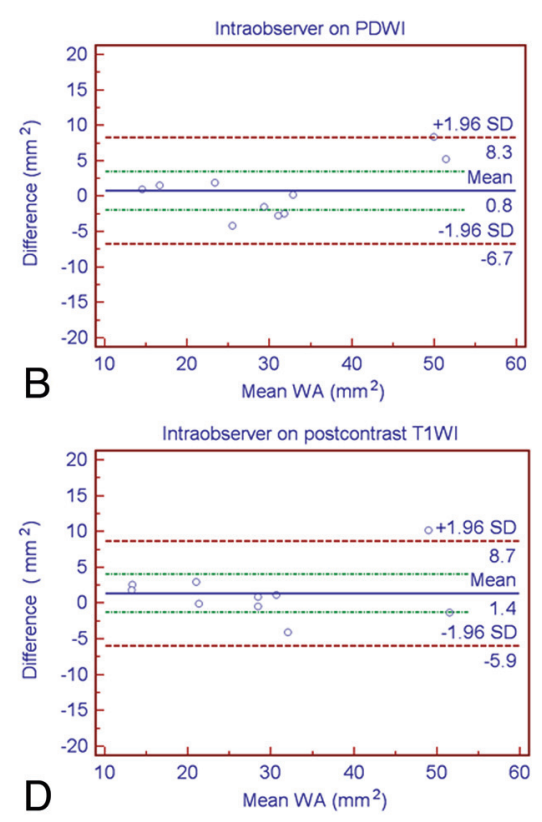

Fig 2. Bland-Altman plots of intraobserver wall area measurements at the level of the trigeminal ganglion on T1WI $(A), \mathrm{PDWI}(B), \mathrm{T} 2 \mathrm{WI}(C)$, and postcontrast T1WI $(D)$ 

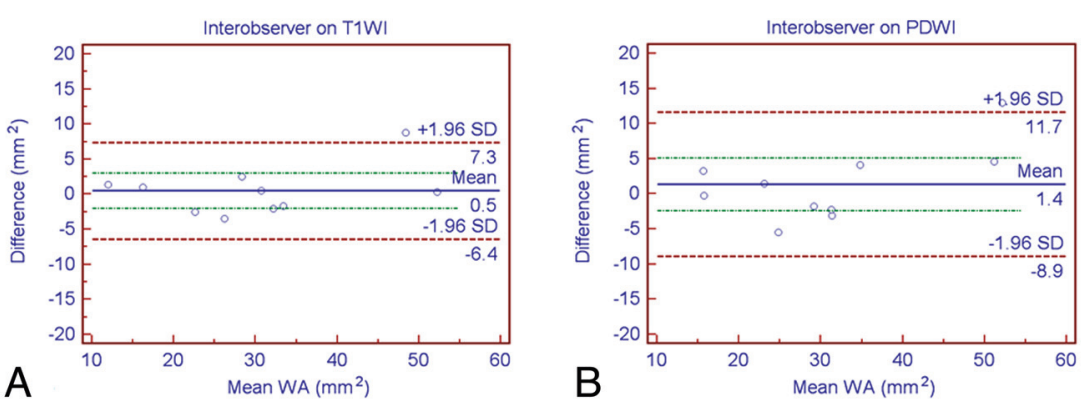

Fig 3. Bland-Altman plots of interobserver wall area measurements at the level of the trigeminal ganglion on T1WI $(A)$, PDWI $(B), \mathrm{T} 2 \mathrm{WI}(C)$, and postcontrast T1WI $(D)$.
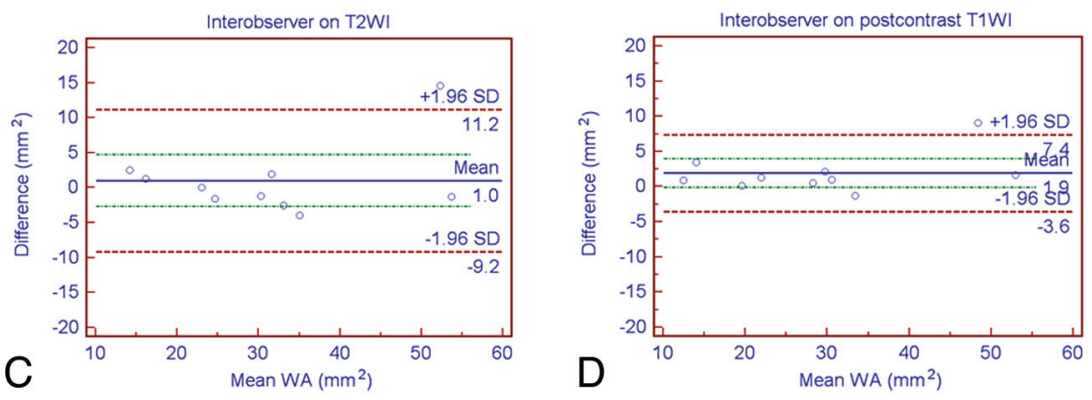

\section{Data Analysis}

The ICC and the lower and upper limits the of the 95\% CI were determined for intraobserver and interobserver variability. The Bland-Altman method for comparing paired measurements on T1WI, PDWI, T2WI, and postcontrast T1WI was used to determine intraobserver and interobserver agreement.

\section{Results}

Between June 2008 and July 2009, 10 consecutive male patients with symptomatic BA atherosclerosis underwent $3 \mathrm{~T}$ HRMRI. Their mean age was 55 years (range, $45-72$ years). All patients had $2(n=5)$ or more $(n=5)$ atherosclerotic risk factors. The qualifying event was posterior circulation ischemic stroke in 1 and TIA in 9 within 90 days.

Image-quality ratings of all images were adequate-to-good. Thus, WA measurements of the BA at the level of trigeminal ganglion were available for analysis in all patients (Fig 1).

The intraobserver variability was small for measuring WA on T1WI, PDWI, T2WI, and postcontrast T1WI, with the ICC ranging from 0.973 to 0.980 (Table). The interobserver variability was also small on these sequences, with the ICC ranging from 0.957 to 0.989 (Table).

The Bland-Altman plot showed strongly agreeing intraobserver (Fig 2) and interobserver (Fig 3) measurements on T1WI, PDWI, T2WI, and postcontrast T1WI.

\section{Discussion}

This study demonstrates that HRMRI can be used to measure the plaque burden of BA atherosclerosis at the level of trigeminal ganglion with high reproducibility. For this pilot study, we chose the level at the trigeminal ganglion for its easy identification on MR images so that the measurements could be obtained as consistently as possible.

Postcontrast T1WI yields better reproducibility for WA measurements than T1WI, PDWI, and T2WI because the outer wall boundary is more clearly delineated. This is similar to previous findings in a carotid atherosclerosis study, ${ }^{8}$ because the arterial wall, including the adventitia layer, is better penetrated by gadolinium-based contrast agents marking the outer wall boundary. To help delineate the vessel wall on PDWI and T2WI sequences, flow compensation and the inferior spatial saturation band were used in our study to suppress the inflow blood signal intensity and to minimize pulsation artifacts. Although the intraobserver and interobserver variability was greater on PDWI and T2WI sequences, it was still acceptable. However, these sequences have the advantage of requiring no contrast and less scanning time. Although obvious calcium in the vessel wall was not encountered in our patients, manually tracing the vessel area is difficult when calcium is present, especially on TIWI, because both CSF and calcium are hypointense. PDWI and T2WI may be more useful for distinguishing the vessel lumen in the presence of calcified plaque because CSF is hyperintense and calcium is hypointense.

HRMRI can potentially be used to monitor the progression or regression of $\mathrm{BA}$ atherosclerotic plaque, but there are some limitations to this study. First, despite our intention to obtain a precise and consistent measurement site by using the trigeminal ganglion as a prominent landmark, some discrepancies on follow-up imaging could not be entirely ruled out due to small size vessel and tortuosity. Second, only a focal site of BA plaque was measured, and it might not be representative of the changes in lesion burden for the rest of the vessel. 3D MR imaging measurements of the BA wall volume may overcome both of these limitations. Third, the appearance of a plaque component on different MR imaging sequences was not analyzed in our current studies and should be further investigated in the future.

\section{Conclusions}

Measurement of the WA in an atherosclerotic BA at the level of the trigeminal ganglion by HRMRI is reproducible. This imaging technique can potentially be used in future studies to monitor the effects of therapeutic intervention on BA plaque progression or regression. 


\section{References}

1. Zhang S, Cai J, Luo Y, et al. Measurement of carotid wall volume and maximum area with contrast-enhanced 3D MR imaging: initial observations. Radiology 2003;228:200-05

2. Mintz GS, Nissen SE, Anderson WD, et al. American College of Cardiology Clinical Expert Consensus Document on Standards for Acquisition, Measurement and Reporting of Intravascular Ultrasound Studies (IVUS): a report of the American College of Cardiology Task Force on Clinical Expert Consensus Documents. J Am Coll Cardiol 2001;37:1478-92

3. Leber AW, Knez A, von Ziegler F, et al. Quantification of obstructive and nonobstructive coronary lesions by 64-slice computed tomography: a comparative study with quantitative coronary angiography and intravascular ultrasound. J Am Coll Cardiol 2005;46:147-54
4. Pignoli P, Tremoli E, Poli A, et al. Intimal plus medial thickness of the arterial wall: a direct measurement with ultrasound imaging. Circulation 1986; 74:1399-406

5. Yuan C, Beach KW, Smith LH Jr, et al. Measurement of atherosclerotic carotid plaque size in vivo using high resolution magnetic resonance imaging. $\mathrm{CirCu}$ lation 1998;98:2666-71

6. Klein IF, Lavallée PC, Touboul PJ, et al. High-resolution MRI identifies basilar artery plaques in paramedian pontine infarct. Neurology 2005;64:551-52

7. Swartz RH, Bhuta SS, Farb RI, et al. Intracranial arterial wall imaging using high-resolution 3-Tesla contrast-enhanced MRI. Neurology 2009;72:627-34

8. Yuan C, Kerwin WS, Ferguson MS, et al. Contrast-enhanced high resolution MRI for atherosclerotic carotid artery tissue characterization. J Magn Reson Imaging 2002;15:62-67 